\title{
ON SOME CONJECTURES ABOUT FREE AND NEARLY FREE DIVISORS
}

\author{
E. ARTAL BARTOLO, L. GORROCHATEGUi, I. LUENGO, AND A. MELLE-HERNÁNDEZ \\ Dedicated to Gert-Martin Greuel on the occasion of his 70th birthday
}

\begin{abstract}
In this paper infinite families of examples of irreducible free and nearly free curves in the complex projective plane which are not rational curves and whose local singularites can have an arbitrary number of branches are given. All these examples answer negatively to some conjectures proposed by A. Dimca and G. Sticlaru. Our examples say nothing about the most remarkable conjecture by A. Dimca and G. Sticlaru, i.e. every rational cuspidal plane curve is either free or nearly free.
\end{abstract}

\section{INTRODUCTION}

The notion of free divisor was introduced by K. Saito [21] in the study of discriminants of versal unfoldings of germs of isolated hypersurface singularites. Since then many interesting and unexpected applications to Singularity Theory and Algebraic Geometry has been appearing. In this paper we are mainly focused on complex projective plane curves and we adapt the corresponding notions and results to this set-up. The results contained in this paper have needed a lot of computations in order to get the right statements. All of them have been done using the computer algebra system Singular [9] through Sagemath [24]. We thanks Singular's team for such a great mathematical tool and specially to Gert-Martin for his dedication to Singular developpement.

Date: October 8, 2018.

2010 Mathematics Subject Classification. 14A05, 14R15.

Key words and phrases. free divisors, nearly free curves.

The first author is partially supported by the Spanish grant MTM2013-45710-C02-01-P and Grupo Geometría of Gobierno de Aragón/Fondo Social Europeo. The last three authors are partially supported by the Spanish grant MTM2013-45710-C02-02-P. 
Let $S:=\mathbb{C}[x, y, z]$ be the polynomial ring endowed with the natural graduation $S=\bigoplus_{m=0}^{\infty} S_{m}$ by homogeneous polynomials. Let $f \in S_{d}$ be a homogeneous polynomial of degree $d$ in the polynomial ring and let $C \subset \mathbb{P}^{2}$ defined by $f=0$ and assume that $C$ is reduced. We denote by $J_{f}$ the Jacobian ideal of $f$, i.e. the homogeneous ideal in $S$ spanned by $f_{x}, f_{y}, f_{z}$, and by $M(f)=S / J_{f}$ the corresponding graded ring, called the Jacobian (or Milnor) algebra of $f$.

Let $I_{f}$ be the saturation of the ideal $J_{f}$ with respect to the maximal ideal $(x, y, z)$ in $S$ and let $N(f)=I_{f} / J_{f}$ be the corresponding graded quotient. Recall that the curve $C: f=0$ is called a free divisor if $N\left((f)=I_{f} / J_{f}=0\right.$, see e.g. [23].

A. Dimca and G. Sticlaru introduced in [14] the notion of nearly free divisor which is a sligth modification of the notion of free divisor. The curve $C$ is called nearly free divisor if $N(f) \neq 0$ and $\operatorname{dim} N(f)_{k} \leq 1$ for any $k$.

The main results in [13, 14] and many infinite families of examples motivate the following conjecture.

Conjecture 1.1. [14]

(i) Any rational cuspidal curve $C$ in the plane is either free or nearly free.

(ii) An irreducible plane curve $C$ which is either free or nearly free is rational.

In [14, the authors provide some interesting results supporting the statement of Conjecture 1.1)(i); in particular, Conjecture 1.1](i) holds for rational cuspidal curves of even degree [14, Theorem 4.1]. They need a topological assumption on the cusps which is not fulfilled all the time when the degree is odd, see [14, Theorem 4.1].

They proved also that this conjecture holds for a curve $C$ with an abelian fundamental group $\pi_{1}\left(\mathbb{P}^{2} \backslash C\right)$ or having as degree a prime power, see [14, Corollary 4.2] and the discussion in [2]. Moreover, any unicuspidal rational curve with a unique Puiseux pair is either free or nearly free, see [14, Corollary 4.5], except the curves of odd degree in one case of the classification of such unicuspidal curves obtained in [15].

As for Conjecture 1.1](ii), note that reducible nearly free curves may have irreducible components which are not rational, see [14, Example 2.8]: a smooth cubic with three tangents at aligned inflection points is nearly free (by the way, the condition of alignment can be removed, at least in some examples computed using [9]). For free curves, examples can be found using [29, Theorem 2.7] e.g. $\left(x^{3}-y^{3}\right)\left(y^{3}-z^{3}\right)\left(x^{3}-z^{3}\right)\left(a x^{3}+b y^{3}+c z^{3}\right)$ for generic $a, b, c \in \mathbb{C}$ such that 
$a+b+c=0$. The conjectures in [29] give some candidate examples in less degree; it is possible to check that $\left(y^{2} z-x^{3}\right)\left(y^{2} z-x^{3}-z^{3}\right)=0$ is free (also compued with [9]). Dimca and Sticlaru also proposed the following conjecture.

Conjecture 1.2. [14]

(i) Any free irreducible plane curve $C$ has only singularities with at most two branches.

(ii) Any nearly free irreducible plane curve $C$ has only singularities with at most three branches.

In this paper we give some examples of irreducible free and nearly free curves in the complex projective plane which are not rational curves giving counterexamples to Conjecture 1.1.(ii). In the same set up, some examples of irreducible free curve whose two singular points has any odd number of branches giving counterexamples Conjecture 1.2] (i) and an irreducible nearly free curve with just one singular point which has 4 branches giving counterexamples Conjecture 1.2](ii) are provided too.

Section 2 is devoted to collect well known results in the theory of free divisors and nearly free divisors mainly form the original papers of A. Dimca and G. Sticlaru [13, 14]. Also a characterization for being nearly-free reduced plane curve from A. Dimca in [10] is also recorded. This characterization is similar to the characterization of being free by du Plessis and Wall in [20].

From Section 3.2 it can be deduced that, for every odd integer $k \geq 1$, the irreducible plane curve $C_{5 k}$ of degree $d=5 k$ defined by

$$
C_{5 k}: f_{5 k}:=\left(y^{k} z^{k}-x^{2 k}\right)^{2} y^{k}-x^{5 k}=0,
$$

has geometric genus equals $g\left(C_{5 k}\right)=\frac{(k-1)(k-2)}{2}$, it has only two singular points and the number of branches of $C_{5 k}$ at each of them is exactly $k$, and moreover it is a free divisor, see Theorem 3.9, This is a counterexample to both the free part of Conjecture 1.1.(ii) and Conjecture 1.2)(i).

From Section 3.3 it can also be deduced that, for any odd integer $k \geq 1$, the irreducible plane curve $C_{4 k}$ of degree $d=4 k$ defined by

$$
C_{4 k}: f_{4 k}:=\left(y^{k} z^{k}-x^{2 k}\right)^{2}-x^{3 k} y^{k}=0,
$$

has genus equals $g\left(C_{4 k}\right)=\frac{(k-1)(k-2)}{2}$, its singular set consists of two singular points and the number of branches of $C_{4 k}$ at each of them is $k$ and now $C_{4 k}$ is a nearly 
free divisor, see Theorem 3.11. This is a counterexample to both the nearly free part of Conjecture 1.1] (ii) and Conjecture 1.2](ii) too.

In the familes studied before the number of singular points of the curves is exactly two. In Section 3.4, we are looking for curves giving a counterexample to the nearly free part of Conjecture 1.1][(ii) with unbounded genus and number of singularities. In particular, for every odd integer $k \geq 1$, the irreducible curve $C_{2 k}$ of degree $d=2 k$ defined by

$$
C_{2 k}: f_{2 k}:=x^{2 k}+y^{2 k}+z^{2 k}-2\left(x^{k} y^{k}+x^{k} z^{k}+y^{k} z^{k}\right)=0
$$

has genus equals $g\left(C_{2 k}\right)=\frac{(k-1)(k-2)}{2}$, its singular set $\operatorname{Sing}\left(C_{2 k}\right)$ consists of exactly $3 k$ singular points of type $\mathbb{A}_{k-1}$ and it is a nearly free divisor, see Theorem 3.12 .

One of the main tools to find such examples is the use of Kummer covers. A Kummer cover is a map $\pi_{k}: \mathbb{P}^{2} \rightarrow \mathbb{P}^{2}$ given by

$$
\pi_{k}([x: y: z]):=\left[x^{k}: y^{k}: z^{k}\right] .
$$

Since Kummer covers are finite Galois unramified covers of $\mathbb{P}^{2} \backslash\{x y z=0\}$ with $\operatorname{Gal}\left(\pi_{k}\right) \cong \mathbb{Z} / k \mathbb{Z} \times \mathbb{Z} / k \mathbb{Z}$, Kummer covers are a very useful tool in order to construct complicated algebraic curves starting from simple ones. We meanly refer to [4, §5] for a systematic study of Kummer covers.

In particular, these familes of examples $\left\{C_{5 k}\right\}$ (which are free), $\left\{C_{4 k}\right\}$ and $\left\{C_{2 k}\right\}$ (which are nearly free) are constructed as the pullback under the Kummer cover $\pi_{k}$ of the corresponding rational cuspidal curves: the quintic $C_{5}$ which is a free divisor, and the corresponding nearly free divisors defined by the cuartic $C_{4}$ and the conic $C_{2}$.

In Section 4, an irreducible nearly free curve of degree 49 which is rational, it has just one singular point which has 4 branches. It is a general element of the unique pencil associated to any rational unicuspidal plane curve see [8].

\section{Free and nearly free Plane Curves after Dimca and Sticlaru}

Let $S:=\mathbb{C}[x, y, z]$ be the polynomial ring endowed with the natural graduation $S=\bigoplus_{m=0}^{\infty} S_{m}$ by homogeneous polynomials. Let $f \in S_{d}$ be a homogeneous polynomial of degree $d$ in the polynomial ring Let $C$ be the plane curve in $\mathbb{P}^{2}$ defined by $f=0$ and assume that $C$ is reduced. We have denoted by $J_{f}$ the Jacobian ideal of $f$, i.e. the homogeneous ideal in $S$ spanned by $f_{x}, f_{y}, f_{z}$. Let 
$M(f)=S / J_{f}$ be the corresponding graded ring, called the Jacobian (or Milnor) algebra of $f$.

The minimal degree of a Jacobian relation for $f$ is the integer $\operatorname{mdr}(f)$ defined to be the smallest integer $m \geq 0$ such that there is a nontrivial relation

$$
a f_{x}+b f_{y}+c f_{z}=0, \quad(a, b, c) \in S_{m}^{3} \backslash(0,0,0) .
$$

When $\operatorname{mdr}(f)=0$, then $C$ is a union of lines passing through one point, a situation easy to analyse. We assume from now on that $\operatorname{mdr}(f) \geq 1$.

\subsection{Free plane curves.}

We have denoted by $I_{f}$ the saturation of the ideal $J_{f}$ with respect to the maximal ideal $(x, y, z)$ in $S$. Let $N(f)=I_{f} / J_{f}$ be the corresponding homogeneous quotient ring.

Consider the graded $S$-submodule

$$
\operatorname{AR}(f)=\left\{(a, b, c) \in S^{3} \mid a f_{x}+b f_{y}+c f_{z}=0\right\} \subset S^{3}
$$

of all relations involving the derivatives of $f$, and denote by $\operatorname{AR}(f)_{m}$ its homogeneous part of degree $m$.

Notation 2.1. We set $\operatorname{ar}(f)_{k}=\operatorname{dim} \mathrm{AR}(f)_{k}, m(f)_{k}=\operatorname{dim} M(f)_{k}$ and $n(f)_{k}=$ $\operatorname{dim} N(f)_{k}$ for any integer $k$.

We use the definition of freeness given by Dimca [10].

Definition 2.2. The curve $C: f=0$ is a free divisor if the following equivalent conditions hold.

(1) $N(f)=0$, i.e. the Jacobian ideal is saturated.

(2) The minimal resolution of the Milnor algebra $M(f)$ has the following form

$$
0 \rightarrow S\left(-d_{1}-d+1\right) \oplus S\left(-d_{2}-d+1\right) \rightarrow S^{3}(-d+1) \stackrel{\left(f_{x}, f_{y}, f_{z}\right)}{\longrightarrow} S
$$

for some positive integers $d_{1}, d_{2}$.

(3) The graded $S$-module $\operatorname{AR}(f)$ is free of rank 2, i.e. there is an isomorphism

$$
\operatorname{AR}(f)=S\left(-d_{1}\right) \oplus S\left(-d_{2}\right)
$$

for some positive integers $d_{1}, d_{2}$. 
When $C$ is a free divisor, the integers $d_{1} \leq d_{2}$ are called the exponents of $C$. They satisfy the relations

$$
d_{1}+d_{2}=d-1 \text { and } \tau(C)=(d-1)^{2}-d_{1} d_{2},
$$

where $\tau(C)$ is the total Tjurina number of $C$, see for instance [11, 13]. Following the deformations results in [23], Sticlaru [25] defines a curve $C \subset \mathbb{P}^{2}$ to be projectively rigid if $\left(I_{f}\right)_{d}=\left(J_{f}\right)_{d}$. In particular, if $C$ is free then it is projectively rigid.

Remark 2.3. This notion of projectively rigid differs from the classical one, see e.g. [16], where a curve is projectively rigid if its equisingular moduli space is discrete. Note that four lines passing through a point define a free divisor but its equisingular moduli space is defined by the cross-ratio.

\subsection{Nearly free plane curves.}

Dimca and Sticlaru introduced the notion of a nearly free divisor which is more subtle, see [14], or the curve version of [12, Remark 5.2 and Theorem 5.3].

Definition 2.4. [14] The curve $C: f=0$ is a nearly free divisor if the following equivalent conditions hold.

(1) $N(f) \neq 0$ and $n(f)_{k} \leq 1$ for any $k$.

(2) The Milnor algebra $M(f)$ has a minimal resolution of the form

$$
0 \rightarrow S\left(-d-d_{2}\right) \rightarrow S\left(-d-d_{1}+1\right) \oplus S^{2}\left(-d-d_{2}+1\right) \rightarrow S^{3}(-d+1) \stackrel{\left(f_{0}, f_{1}, f_{2}\right)}{\longrightarrow} S
$$

for some integers $1 \leq d_{1} \leq d_{2}$, called the exponents of $C$.

(3) There are 3 syzygies $\rho_{1}, \rho_{2}, \rho_{3}$ of degrees $d_{1}, d_{2}=d_{3}=d-d_{1}$ which form a minimal system of generators for the first-syzygy module $\operatorname{AR}(f)$.

If $C: f=0$ is nearly free, then the exponents $d_{1} \leq d_{2}$ satisfy

$$
d_{1}+d_{2}=d \text { and } \tau(C)=(d-1)^{2}-d_{1}\left(d_{2}-1\right)-1
$$

see [14]. For both a free and a nearly free curve $C: f=0$, it is clear that $\operatorname{mdr}(f)=d_{1}$.

Remark 2.5. In [14] it is shown that to construct a resolution (2.3) for a given polynomial $f$ one needs the integer $b:=d_{2}-d+2$ and the following ingredients. 
(i) Three syzygies $r_{i}=\left(a_{i}, b_{i}, c_{i}\right) \in S_{d_{i}}^{3}, i=1,2,3$, for $\left(f_{x}, f_{y}, f_{z}\right)$, i.e.

$$
a_{i} f_{x}+b_{i} f_{y}+c_{i} f_{z}=0,
$$

necessary to construct the morphism

$$
\bigoplus_{i=1}^{3} S\left(-d_{i}-(d-1)\right) \rightarrow S^{3}(-d+1), \quad\left(u_{1}, u_{2}, u_{3}\right) \mapsto u_{1} r_{1}+u_{2} r_{2}+u_{3} r_{3}
$$

(ii) One relation $R=\left(v_{1}, v_{2}, v_{3}\right) \in \bigoplus_{i=1}^{3} S\left(-d_{i}-(d-1)\right)_{b+2(d-1)}$ among $r_{1}, r_{2}, r_{3}$, i.e. $v_{1} r_{1}+v_{2} r_{2}+v_{3} r_{3}=0$, necessary to construct the morphism

$$
S(-b-2(d-1)) \rightarrow \bigoplus_{i=1,3} S\left(-d_{i}-(d-1)\right)
$$

by the formula $w \mapsto w R$. Note that $v_{i} \in S_{b-d_{i}+d-1}$.

Corollary 2.6. 14] Let $C: f=0$ be a nearly free curve of degree $d$ with exponents $\left(d_{1}, d_{2}\right)$. Then $N(f)_{k} \neq 0$ for $d+d_{1}-3 \leq k \leq d+d_{2}-3$ and $N(f)_{k}=0$ otherwise. The curve $C$ is projectively rigid if and only if $d_{1} \geq 4$.

\subsection{Characterization of free and nearly free reduced plane curves.}

Just recently Dimca provides in [10] the following characterization of free and nearly free reduced plane curves. For a positive integer $r$, define

$$
\tau(r)_{\max }:=(d-1)(d-r-1)+r^{2} .
$$

Theorem 2.7 ([10]). Let $C \subset \mathbb{P}^{2}$ be a reduced curve of degree d defined by $f=0$, and let $r:=\operatorname{mdr}(f)$.

(1) If $r=<\frac{d}{2}$, then $\tau(C)=\tau(r)_{\max }$ if and only if $C: f=0$ is a free curve.

(2) If $r=\leq \frac{d}{2}$, then $\tau(C)=\tau(r)_{\max }-1$ if and only if $C$ is a nearly free curve.

As it is recalled in [10], Theorem 2.7](1)] is a corollary of [20, Theorem 3.2] by du Plessis and Wall.

\section{High-Genus CURVES Which ARE FREE OR NEARLY FREE DiVisors}

\subsection{Transformations of curves by Kummer covers.}

A Kummer cover is a map $\pi_{k}: \mathbb{P}^{2} \rightarrow \mathbb{P}^{2}$ given by $\pi_{k}([x: y: z]):=\left[x^{k}: y^{k}: z^{k}\right]$. Kummer covers are a very useful tool in order to construct complicated algebraic curves starting from simple ones. Since Kummer covers are finite Galois unramified covers of $\mathbb{P}^{2} \backslash\{x y z=0\}$ with $\operatorname{Gal}\left(\pi_{k}\right) \cong \mathbb{Z} / k \mathbb{Z} \times \mathbb{Z} / k \mathbb{Z}$, topological properties of the new curves can be obtained: Alexander polynomial, fundamental group, 
characteristic varieties and so on (see [1, [3, [5, 28, 17, 6, 4, 18] for papers using these techniques).

Example 3.1. In [28], Uludağ constructs new examples of Zariski pairs using former ones and Kummer covers. He also uses the same techniques to construct infinite families of curves with finite non-abelian fundamental groups.

Example 3.2. In [17, 5], the Kummer covers allow to construct curves with many cusps and extremal properties for their Alexander invariants. These ideas are pushed further in [6] where the authors find Zariski triples of curves of degree 12 with 32 ordinary cusps (distinguished by their Alexander polynomial). Within the same ideas Niels Lindner [18] constructed an example of a cuspidal curve $C^{\prime}$ of degree 12 with 30 cusps and Alexander polynomial $t^{2}-t+1$. For this, he started with a sextic $C_{0}$ with 6 cusps, admitting a toric decomposition. He pulled back $C_{0}$ under a Kummer map $\pi_{2}: \mathbb{P}^{2} \rightarrow \mathbb{P}^{2}$ ramified above three inflectional tangents of $C_{0}$. Since the sextic is of torus type, then same holds for the pullback. Lindner showed that the Mordell-Weil lattice has rank 2 and that the Mordell-Weil group contains $A_{2}(2)$.

A systematic study of Kummer covers of projective plane curves have been done by J.I. Cogolludo, J. Ortigas and the first named author in [4, §5]. Some of results are collected next.

Let $C$ be a (reduced) projective curve of degree $d$ of equation $F_{d}(x, y, z)=0$ and let $\bar{C}_{k}$ be its transform by a Kummer cover $\pi_{k}, k \geq 1$. Note that $\bar{C}_{k}$ is a projective curve of degree $d k$ of equation $F_{d}\left(x^{k}, y^{k}, z^{k}\right)=0$.

Definition 3.3. [4] Let $P \in \mathbb{P}^{2}$ such that $P:=\left[x_{0}: y_{0}: z_{0}\right]$. We say that $P$ is a point of type $\left(\mathbb{C}^{*}\right)^{2}$ (or simply of type 2) if $x_{0} y_{0} z_{0} \neq 0$. If $x_{0}=0$ but $y_{0} z_{0} \neq 0$ the point is said to be of type $\mathbb{C}_{x}^{*}$ (types $\mathbb{C}_{y}^{*}$ and $\mathbb{C}_{z}^{*}$ are defined accordingly). Such points will also be referred to as type 1 points. The corresponding line (either $L_{X}:=\{X=0\}, L_{Y}:=\{Y=0\}$, or $\left.L_{Z}:=\{Z=0\}\right)$ a type-1 lies on will be referred to as their axis. The remaining points $P_{x}:=[1: 0: 0], P_{y}:=[0: 1: 0]$, and $P_{z}:=[0: 0: 1]$ will be called vertices (or type 0 points) and their axes are the two lines (either $L_{X}, L_{Y}$, or $L_{Z}$ ) they lie on.

Remark 3.4. [4] Note that a point of type $\ell, \ell=0,1,2$ in $\mathbb{P}^{2}$ has exactly $k^{\ell}$ preimages under $\pi_{k}$. It is also clear that the local type of $\bar{C}_{k}$ at any two points on 
the same fiber are analytically equivalent. The singularities of $\bar{C}_{k}$ are described in the following proposition.

Proposition 3.5. 4] Let $P \in \mathbb{P}^{2}$ be a point of type $\ell$ and $Q \in \pi_{k}^{-1}(P)$. One has the following:

(1) If $\ell=2$, then $(C, P)$ and $\left(\bar{C}_{k}, Q\right)$ are analytically isomorphic.

(2) If $\ell=1$, then $\left(\bar{C}_{k}, Q\right)$ is a singular point of type 1 if and only if $m>1$, where $m:=(C \cdot \bar{L})_{P}$ and $\bar{L}$ is the axis of $P$.

(3) If $\ell=0$, then $\left(\bar{C}_{k}, Q\right)$ is a singular point.

Remark 3.6. Using Proposition 3.5 (1), if $\operatorname{Sing}(C) \subset\{x y z=0\}$ then $\operatorname{Sing}\left(\bar{C}_{k}\right) \subset$ $\{x y z=0\}$.

Example 3.7. 4] In some cases, we can be more explicit about the singularity type of $\left(\bar{C}_{k}, Q\right)$. If $P$ is of type $1,(C, P)$ is smooth and $m:=(C \cdot \bar{L})_{P}$ then $\left(\bar{C}_{k}, Q\right)$ has the same topological type as $u_{0}^{k}-v_{0}^{m}=0$. In particular, if $m=2$, then $\left(\bar{C}_{k}, Q\right)$ is of type $\mathbb{A}_{k-1}$.

In order to better describe singular points of type 0 and 1 of $\bar{C}_{k}$ we will introduce some notation. Let $P \in \mathbb{P}^{2}$ be a point of type $\ell=0,1$ and $Q \in \pi_{k}^{-1}(P)$ a singular point of $\bar{C}_{k}$. Denote by $\mu_{P}$ (resp. $\mu_{Q}$ ) the Milnor number of $C$ at $P$ (resp. $\bar{C}_{k}$ at $Q$ ). Since $\ell=0,1$, then $P$ and $Q$ belong to either exactly one or two axes. If $P$ and $Q$ belong to an axis $\bar{L}$, then $m_{P}^{\bar{L}}:=(C \cdot \bar{L})_{P}$ (analogous notation for $Q$ ). More specific details about singular points of types 0 and 1 can be described as follows.

Proposition 3.8. [4] Under the above conditions and notation one has the following

(1) For $\ell=1, P$ belongs to a unique axis $\bar{L}$ and

(a) $\mu_{Q}=k \mu_{P}+\left(m_{P}^{\bar{L}}-1\right)(k-1)$,

(b) If $(C, P)$ is locally irreducible and $r:=\operatorname{gcd}\left(k, m_{P}^{\bar{L}}\right)$, then $(C, Q)$ has $r$ irreducible components which are analytically isomorphic to each other.

(2) For $\ell=0, P$ belongs to exactly two axes $\bar{L}_{1}$ and $\bar{L}_{2}$

(a) $\mu_{Q}=k^{2}\left(\mu_{P}-1\right)+k(k-1)\left(m_{P}^{\bar{L}_{1}}+m_{P}^{\bar{L}_{2}}\right)+1$ (There is a typo in the printed formula in [4]: this one is obtained by adding $\left.k-k^{2}\right)$. 
(b) If $(C, P)$ is locally irreducible and $r:=\operatorname{gcd}\left(k, m_{P}^{\bar{L}_{1}}, m_{P}^{\bar{L}_{2}}\right)$, then $(C, Q)$ has $\mathrm{kr}$ irreducible components which are analytically isomorphic to each other.

\subsection{Irreducible free curves with many branches and high genus.}

Let us consider the quintic curve $C_{5}$, see Figure 1, defined by $f_{5}:=(y z-$ $\left.x^{2}\right)^{2} y-x^{5}=0$. It has two singular points, $p_{1}=[0: 1: 0]$ of type $\mathbb{A}_{4}$ and $p_{2}=[0: 0: 1]$ of type $\mathbb{E}_{8}$, so that it is rational and cuspidal. This curve is free, see [13, Theorem 4.6]. Let us consider the Kummer cover $\pi_{k}: \mathbb{P}^{2} \rightarrow \mathbb{P}^{2}$ given by $\pi_{k}([x: y: z]):=\left[x^{k}: y^{k}: z^{k}\right]$ and its Kummer transform $C_{5 k}$, defined by $f_{5 k}:=\left(y^{k} z^{k}-x^{2 k}\right)^{2} y^{k}-x^{5 k}=0$.

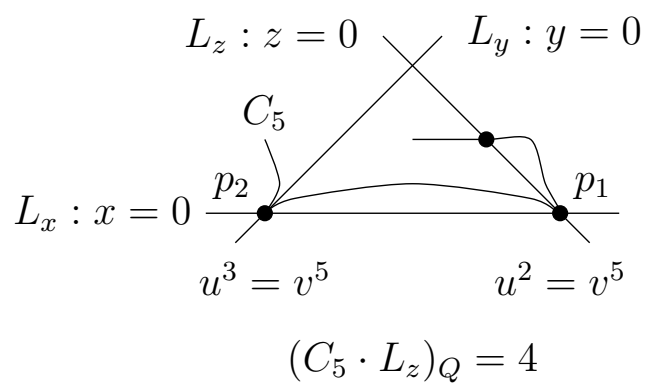

Figure 1. Curve $C_{5}$

Theorem 3.9. For any $k \geq 1$, the curve $C_{5 k}$ of degree $d=5 k$ defined by

$$
C_{5 k}: f_{5 k}:=\left(y^{k} z^{k}-x^{2 k}\right)^{2} y^{k}-x^{5 k}=0,
$$

verifies the following properties:

(1) $\operatorname{Sing}\left(C_{5 k}\right)=\left\{p_{1}, p_{2}\right\}$. The number of branches of $C_{5 k}$ at $p_{2}$ is $k$, and at $p_{1}$, it equals $k$ (if $k$ is odd) or $2 k$ (if $k$ is even).

(2) $C_{5 k}$ is a free divisor with exponents $d_{1}=2 k, d_{2}=3 k-1$ and $\tau\left(C_{5 k}\right)=$ $19 k^{2}-8 k+1$.

(3) $C_{5 k}$ has two irreducible components of genus $\frac{(k-2)^{2}}{4}$ if $k$ is even and irreducible of genus $\frac{(k-1)(k-2)}{2}$ otherwise.

Proof. Part (1) is an easy consequence of in [4, Lemma 5.3, Proposition 5.4 and Proposition 5.6]. The singularites $\operatorname{Sing}\left(C_{5}\right)=\left\{p_{1}, p_{2}\right\}$ are of type 0 , in the sense of the Kummer cover $\pi_{k}$ (see Definition [3.3) and $C_{5}$ has no singularities outside the intersection points of the axes. Moreover $C_{5}$ intersects the line $L_{z}$ transversally at 
a point of type 1; then by Proposition 3.5 (2) and by Remark 3.6, the singularities of $C_{5 k}$ are exactly the points $p_{1}$ and $p_{2}$.

Since $p_{1}$ and $p_{2}$ are of type 0 we deduce the structure of $C_{5 k}$ at these points. using Proposition 3.8 (2) (b). At $p_{1}$ one has $\left(C_{5}, L_{z}\right)_{p_{1}}=5,\left(C_{5}, L_{x}\right)_{p_{1}}=2$ and $r_{p_{1}}=\operatorname{gcd}(k, 2,5)=1$ for all $k$, and so that the number of branches of $C_{5 k}$ at $p_{1}$ is equal to $k$. In the same way, at $p_{2}$, the intersection $\left(C_{5}, L_{x}\right)_{p_{2}}=2,\left(C_{5}, L_{y}\right)_{p_{2}}=4$ and $r_{p_{2}}=\operatorname{gcd}(k, 2,4)=\operatorname{gcd}(k, 2)$. Ik $k$ is odd, $r_{p_{2}}=1$ and the number of branches of $C_{5 k}$ at $p_{2}$ is equal to $k$. Otherwise $r_{p_{2}}=2$ and the number of branches of $C_{5 k}$ at $p_{2}$ is equal to $2 k$.

In order to prove (2), we follow the ideas of [13, Theorem 4.6]. Let us study first the syzygies of the free curve $C_{5}$. Let us denote by $D_{u, v, w}$, the diagonal matrix with entries $u, v, w$, and define the vectors

$$
R_{1}=\left(0,2 y, x^{2}-3 y z\right), \quad R_{2}=\left(2\left(x^{2}-y z\right), 2\left(5 x^{2}-4 x y+15 y z\right), 8 x-45 z\right)
$$

Let us denote by $J$ the Jacobian ideal $J$ of $f_{5}$. Let us denote by $J_{x}$ the ideal generated by $\left(x f_{5 x}, f_{5 y}, f_{5 z}\right)$. In the same way, we consider the ideals $J_{y}, J_{z}, J_{x y}$, $J_{x z}, J_{y z}, J_{x y z}$. The Table 1 shows bases for the syzygies of these ideals, computed with Singular [9]. Note that

\begin{tabular}{|c|c|c|}
\hline Ideal & First generator & Second generator \\
\hline$J$ & $R_{1} \cdot D_{1, y, 1}$ & $R_{2} \cdot D_{1,1, z}$ \\
\hline$J_{x}$ & $R_{1} \cdot D_{1, y, 1}$ & $R_{2} \cdot D_{1, x, x z}$ \\
\hline$J_{y}$ & $R_{1}$ & $R_{2} \cdot D_{y, 1, y z}$ \\
\hline$J_{z}$ & $R_{1} \cdot D_{1, y z, 1}$ & $R_{2}$ \\
\hline$J_{x y}$ & $R_{1}$ & $R_{2} \cdot D_{y, x, x y z}$ \\
\hline$J_{x z}$ & $R_{1} \cdot D_{1, y z, 1}$ & $R_{2} \cdot D_{1, x, x}$ \\
\hline$J_{y z}$ & $R_{1} \cdot D_{1, z, 1}$ & $R_{2} \cdot D_{y, 1, y}$ \\
\hline$J_{x y z}$ & $R_{1} \cdot D_{1, z, 1}$ & $R_{2} \cdot D_{y, x, x y}$ \\
\hline
\end{tabular}

TABLE 1. Bases of syzygies

$$
f_{5 k x}=k x^{k-1} f_{5 x}\left(x^{k}, y^{k}, z^{k}\right), f_{5 k_{y}}=k y^{k-1} f_{5 y}\left(x^{k}, y^{k}, z^{k}\right), f_{5 k_{z}}=k z^{k-1} f_{5 z}\left(x^{k}, y^{k}, z^{k}\right)
$$


Let $S_{k}:=\mathbb{C}\left[x^{k}, y^{k}, z^{k}\right]$. We have a decomposition

$$
S=\bigoplus_{(i, j, l) \in\{0, \ldots, k-1\}} x^{i} y^{j} z^{l} S_{k}
$$

By construction, $f_{5 k_{x}} \in x^{k-1} S_{k}, f_{5 k_{y}} \in y^{k-1} S_{k}$ and $f_{5 k_{z}} \in z^{k-1} S_{k}$. Hence, in order to compute the syzygies $(a, b, c)$ among the partial derivatives of $f_{5 k}$, we need to characterize the triples $(a, b, c)$ such that each entry belongs to a factor of the decomposition (3.2).

Let us assume that $a \in x^{i_{x}} y^{j_{x}} z^{j_{x}} S_{k}, b \in x^{i_{y}} y^{j_{y}} z^{j_{y}} S_{k}$ and $c \in x^{i_{z}} y^{j_{z}} z^{j_{z}} S_{k}$. We deduce that

$$
i_{x}+k-1 \equiv i_{y} \equiv i_{z} \bmod k \Longrightarrow i=i_{y}=i_{z} \text { and } i_{x}= \begin{cases}i+1 & \text { if } i<k-1 \\ 0 & \text { if } i=k-1 .\end{cases}
$$

Analogous relations hold for the other indices. We distinguish four cases:

Case 1. $i=j=l=k-1$.

In this case $a(x, y, z)=y^{k-1} z^{k-1} \alpha\left(x^{k}, y^{k}, z^{k}\right), b(x, y, z)=x^{k-1} z^{k-1} \beta\left(x^{k}, y^{k}, z^{k}\right)$ and $c(x, y, z)=x^{k-1} y^{k-1} \gamma\left(x^{k}, y^{k}, z^{k}\right)$. Hence $(\alpha, \beta, \gamma)$ is a syzygy for the partial derivatives of $f_{5}$. We conclude that $(a, b, c)$ is a combination of:

$$
R_{1}\left(x^{k}, y^{k}, z^{k}\right) \cdot D_{1, x^{k-1}} y^{k} z^{k-1}, x^{k-1} y^{k-1}=x^{k-1} y^{k-1} R_{1}\left(x^{k}, y^{k}, z^{k}\right) \cdot D_{1, y z^{k-1}, 1}
$$

and

$R_{2}\left(x^{k}, y^{k}, z^{k}\right) \cdot D_{y^{k-1} z^{k-1}, x^{k-1} z^{k-1}, x^{k-1} y^{k-1} z^{k}}=z^{k-1} R_{2}\left(x^{k}, y^{k}, z^{k}\right) \cdot D_{y^{k-1}, x^{k-1}, x^{k-1}} y^{k-1} z$.

Taking out common factors we get syzygies of degree $2 k$ and $3 k-1$.

Case 2. $i<k-1, j=l=k-1$.

In this case $a(x, y, z)=x^{i+1} y^{k-1} z^{k-1} \alpha\left(x^{k}, y^{k}, z^{k}\right), b(x, y, z)=x^{i} z^{k-1} \beta\left(x^{k}, y^{k}, z^{k}\right)$ and $c(x, y, z)=x^{i} y^{k-1} \gamma\left(x^{k}, y^{k}, z^{k}\right)$. Hence $(\alpha, \beta, \gamma)$ is a syzygy for the generators of the ideal $J_{x}$. It is easily seen that we obtain combination of generators of the above syzygies. The other cases are treated in the same way.

We conclude that $C_{5 k}$ is free with $d_{1}=\operatorname{mdr}\left(f_{5 k}\right)=2 k$ and $d_{2}=d-1-d_{1}=$ $5 k-1-2 k=3 k-1$. By equation (2.2) $\tau\left(C_{5 k}\right)=19 k^{2}-8 k+1$ for all $k$.

In order to prove $(3)$, we study the branched cover $\tilde{\pi}_{k}: \tilde{C}_{5 k} \rightarrow \tilde{C}_{5}$ between the normalizations of the curves. The monodromy of this map as an unramified cover 
of $\mathbb{P}^{2} \backslash\{x y z=0\}$ is determined by an epimorphism

$$
H_{1}\left(\mathbb{P}^{2} \backslash\{x y z=0\} ; \mathbb{Z}\right) \rightarrow \mathbb{Z}_{k} \times \mathbb{Z}_{k}=: G_{k}
$$

such that the meridians of the lines are sent to $a_{x}, a_{y}, a_{z}$, a system of generators of $G_{k}$ such that $a_{x}+a_{y}+a_{z}=0$. Since the singularities of $C_{5}$ are locally irreducible, then $C_{5}$ and $\tilde{C}_{5}$ are homeomorphic, and the covering $\tilde{\pi}_{k}$ is determined by the monodromy map

$$
H_{1}\left(\tilde{C}_{5} \backslash\{x y z=0\} ; \mathbb{Z}\right) \rightarrow \mathbb{Z}_{k} \times \mathbb{Z}_{k}=: G_{k}
$$

obtained by composing with the map defined by the inclusion. Hence $\tilde{C}_{5} \backslash\{x y z=$ $0\}$ is isomorphic to $\mathbb{P}^{1} \backslash\{$ three points $\}$ ). The image of a meridian corresponding to a point $P$ in the axes is given by

$$
m_{P}^{L_{x}} a_{x}+m_{P}^{L_{y}} a_{y}+m_{P}^{L_{z}} a_{z} .
$$

Hence, we obtain $a_{z}$ (the smooth point), $3 a_{x}+5 a_{y}$ (the $\mathbb{E}_{8}$-point) and $2 a_{x}+4 a_{z}$ (the $\mathbb{A}_{4}$-point). In terms of the basis $a_{y}, a_{z}$ they read as $a_{z}, 2 a_{y}-3 a_{z},-2 a_{y}+2 a_{z}$, i.e., the monodromy group is generated by $2 a_{y}, a_{z}$. If $k$ is pair, the monodromy group is of index 2 in $G_{k}$, and hence $\tilde{C}_{5 k}$ has two connected components, while it is $G_{k}$ when $k$ is odd and $\tilde{C}_{5 k}$ is connected. These properties give us the statement about the number of irreducible components.

The genus can be computed using the singularities of $C_{5 k}$ or via RiemannHurwitz's formula. Note that the covering $\tilde{\pi}_{k}$ is of degree $k^{2}$ with three ramification points; at $p_{2}$ and the smooth point in the axis we find $k$ preimages, while at $p_{1}$ we find $k$ preimages if $k$ is odd and $2 k$ preimages if it is even, because of (1). Hence, for $k$ odd

$$
\chi\left(\tilde{C}_{5 k}\right)=-k^{2}+3 k \Longrightarrow g\left(\tilde{C}_{5 k}\right)=\frac{(k-1)(k-2)}{2}
$$

and for $k$ even, where $\tilde{C}_{5 k}=\tilde{C}_{5 k}^{1} \cup \tilde{C}_{5 k}^{2}$,

$$
\chi\left(\tilde{C}_{5 k}\right)=-k^{2}+4 k \Longrightarrow g\left(\tilde{C}_{5 k}^{i}\right)=\frac{2-\frac{\chi\left(\tilde{C}_{5 k}\right)}{2}}{2}=\frac{(k-2)^{2}}{4} .
$$

So, for odd $k \geq 3$, the curve $C_{5 k}$ is an irreducible free curve of positive genus whose singularities have $k$ branches each. This is a counterexample to both the free part of Conjecture 1.1](ii) and Conjecture 1.2](i).

Remark 3.10. Up to projective transformation, there are two quintic curves with two singular points of type $\mathbb{A}_{4}$ and $\mathbb{E}_{8}$. One is $C_{5}:\left(y z-x^{2}\right)^{2} y-x^{5}=0$, which 
is free; there is another one $D_{5}: g=y^{3} z^{2}-x^{5}=0$ (the contact of the tangent line to the $\mathbb{A}_{4}$-point distinguishes both curves). The curve $D_{5}$ is nearly free; it is easily seen that $\operatorname{mdr}(g)=1$. Since both singular points are quasihomogeneous, $\tau(C)=12$, and we may apply Theorem 2.7] $(2)$ the pair $\left(C_{5}, \tilde{C}_{5}\right)$ is a kind of counterexample to Terao's conjecture [19, Conjecture 4.138] for irreducible divisors (with constant Tjurina number), compare with [22].

\subsection{Irreducible nearly free curves with many branches and high genus.}

The quartic curve $C_{4}$ defined by $f_{4}:=\left(y z-x^{2}\right)^{2}-x^{3} y=0$ has two singular points, $p_{1}=[0: 1: 0]$ of type $\mathbb{A}_{2}$ and $p_{2}=[0: 0: 1]$ of type $\mathbb{A}_{4}$, in particular it is rational and cuspidal. We will consider the Kummer transform $C_{4 k}$, defined by $f_{4 k}:=\left(y^{k} z^{k}-x^{2 k}\right)^{2}-x^{3 k} y^{k}=0$, of the curve $C_{4}$.

Theorem 3.11. For any $k \geq 1$, the curve $C_{4 k}$ of degree $d=4 k$ defined by

$$
C_{4 k}: f_{4 k}:=\left(y^{k} z^{k}-x^{2 k}\right)^{2}-x^{3 k} y^{k}=0,
$$

verifies the following properties

(1) $\operatorname{Sing}\left(C_{4 k}\right)=\left\{p_{1}, p_{2}\right\}$. The number of branches of $C_{4 k}$ at each $p_{2}$ is $k$, and at $p_{1}$, it equals $k$ (if $k$ is odd) or $2 k$ (if $k$ is even).

(2) $C_{4 k}$ is a nearly free divisor with exponents $d_{1}=d_{2}=d_{3}=2 k$ and $\tau\left(C_{4 k}\right)=$ $6 k(2 k-1)$.

(3) $C_{4 k}$ has two irreducible components of genus $\frac{(k-2)^{2}}{4}$ if $k$ is even and irreducible of genus $\frac{(k-1)(k-2)}{2}$ otherwise.

Proof. Since $\operatorname{Sing}\left(C_{4}\right)\left(=\left\{p_{1}, p_{2}\right\}\right)$ are points of type $0, C_{4}$ meets $\{x y z=0\}$ at three points $p_{1}, p_{2}$ and transversally at $p_{3}$ which is of type 1 then $\operatorname{Sing}\left(C_{4 k}\right)=\left\{p_{1}, p_{2}\right\}$ and to prove Part (1) its enough to find the number of branches of $C_{4 k}$ at these points using Proposition 3.8 (2) (b). At $p_{1}$ one has $\left(C_{4}, L_{z}\right)_{p_{1}}=3,\left(C_{4}, L_{x}\right)_{p_{1}}=2$ and $r_{p_{1}}=\operatorname{gcd}(k, 2,3)=1$ for all $k$, and so that the number of branches of $C_{4 k}$ at $p_{1}$ is equal to $k$. In the same way, at $p_{2}$, the intersection $\left(C_{4}, L_{x}\right)_{p_{2}}=2$, $\left(C_{4}, L_{y}\right)_{p_{2}}=4$ and $r_{p_{2}}=\operatorname{gcd}(k, 2,4)=\operatorname{gcd}(k, 2)$. Ik $k$ is odd, $r_{p_{2}}=1$ and the number of branches of $C_{4 k}$ at $p_{2}$ is equal to $k$. Otherwise $r_{p_{2}}=2$ and the number of branches of $C_{4 k}$ at $p_{2}$ is equal to $2 k$.

The proof of Part (2) follows the same guidelines as in Theorem 3.9. With the notations of that proof, a generator system for the syzygies of $J$ (Jacobian ideal 
of $\left.f_{4}\right)$ is given by:

$$
\begin{aligned}
& R_{1}:=(y(3 x-4 z), 3 y(4 x-3 y), z(9 y-20 x)), \\
& R_{2}:=\left(-x(x+2 z),-4 x^{2}+3 x y+10 y z,-z(3 x+10 z)\right), \\
& R_{3}:=\left(x y,-3 y^{2}, 2 x^{2}+3 y z\right) .
\end{aligned}
$$

These syzygies satisfy the relation $x R_{1}+3 y R_{2}+10 z R_{3}=0$. And by Dimca Steclaru Remark (2.5) $C_{4}$ is a nearly free divisor with exponents $d_{1}=d_{2}=d_{3}=2$.

For the ideal $J_{z}$, we have a similar situation. For the other ideals, their syzygy space is free of rank 2 . Using these results it is not hard to prove that the syzygies of $f_{4 k}$ are generated by

$$
\begin{aligned}
& R_{k, 1}:=\left(y^{k}\left(3 x^{k}-4 z^{k}\right), 3 x^{k-1} y\left(4 x^{k}-3 y^{k}\right), x^{k-1} z\left(9 y^{k}-20 x^{k}\right)\right), \\
& R_{k, 2}:=\left(-x y^{k-1}\left(x^{k}+2 z^{k}\right),-4 x^{2 k}+3 x^{k} y^{k}+10 y^{k} z^{k},-y^{k-1} z\left(3 x^{k}+10 z^{k}\right)\right), \\
& R_{k, 3}:=\left(x y^{k} z^{k-1},-3 y^{k+1} z^{k-1}, 2 x^{2 k}+3 y^{k} z^{k}\right) .
\end{aligned}
$$

The results follow as in the proof of Theorem 3.9.

These syzygies satisfy the relation $x R_{k, 1}+3 y R_{k, 2}+10 z R_{k, 3}=0$ and by Dimca Steclaru Remark (2.5) $C_{4 k}$ is a nearly free divisor with exponents $d_{1}=d_{2}=d_{3}=$ $2 k$ and by equation (2.4) $\tau\left(C_{4 k}\right)=6 k(2 k-1)$.

The proof of Part (3) follows the same ideas as in Theorem 3.9 (3).

So, for odd $k \geq 3$, the curve $C_{4 k}$ is an irreducible nearly free curve of positive genus whose singularities have $k$ branches each. This is a counterexample to both the nearly free part of Conjecture 1.1](ii) and Conjecture 1.2](ii).

\subsection{Positive genus nearly-free curves with many singularities.}

Let us consider the conic $C_{2}$ given by $f_{2}=x^{2}+y^{2}+z^{2}-2(x y+x z+y z)=0$. This conic is tangent to three axes and it is very useful to produce interesting curves using Kummer covers.

Theorem 3.12. For any $k \geq 1$, the curve $C_{2 k}$ of degree $d=2 k$ defined by

$$
C_{2 k}: f_{2 k}:=x^{2 k}+y^{2 k}+z^{2 k}-2\left(x^{k} y^{k}+x^{k} z^{k}+y^{k} z^{k}\right)=0,
$$

verifies the following properties

(1) $\operatorname{Sing}\left(C_{2 k}\right)$ are $3 k$ singular points of type $\mathbb{A}_{k-1}$.

(2) $C_{2 k}$ is a nearly free divisor with exponents $d_{1}=d_{2}=d_{3}=k$ and $\tau\left(C_{2 k}\right)=$ $3 k(k-1)$. 


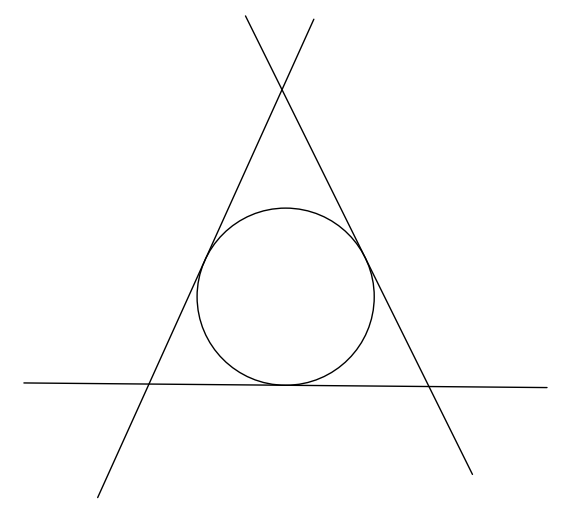

Figure 2. Conic $C_{2}$.

(3) $C_{2 k}$ is irreducible of genus $\frac{(k-1)(k-2)}{2}$ if $k$ is odd and it has four irreducible smooth components of degree $\frac{k}{2}$ if $k$ is even.

Proof. To prove (1) it is enough to take into the account that $C_{2}$ is nonsingular and by Remark 3.6 the singularites of $C_{2 k}$ satisfy $\operatorname{Sing}\left(C_{2 k}\right) \subset\{x y z=0\}$. Moreover $C_{2}$ is tangent to the three axes at 3 points $\left\{p_{1}, p_{2}, p_{3}\right\}$ of type 1 with $\left(C_{2}, L_{x}\right)_{p_{1}}=$ $\left(C_{2}, L_{y}\right)_{p_{2}}=\left(C_{2}, L_{z}\right)_{p_{3}}=2$ at these points. For $i=1, \ldots, 3$, the points $p_{i}$ are of type 1 and by Remark 3.4 all the $k$ preimages under $\pi_{k}$ are analytically equivalent and by Example 3.7 over each $p_{i}$ one has $k$ singular points of type $\mathbb{A}_{k-1}$.

Let us study (2), A generator system for the syzygies of $J$ (Jacobian ideal of $f_{2}$ ) is given by:

$$
\begin{aligned}
& R_{1}:=(y-z, y,-z), \\
& R_{2}:=(-x, z-x, z), \\
& R_{3}:=(x,-y, x-y) .
\end{aligned}
$$

These syzygies satisfy the relation $x R_{1}+y R_{2}+z R_{3}=0$. The other ideals have free 2-rank syzygy modules. An easy computation gives the following syzygies for $f_{2 k}$ :

$$
\begin{aligned}
& R_{k, 1}:=\left(y^{k}-z^{k}, x^{k-1} y,-x^{k-1} z\right), \\
& R_{k, 2}:=\left(-x y^{k-1}, z^{k}-x^{k}, y^{k-1} z\right), \\
& R_{k, 3}:=\left(x z^{k-1},-y z^{k-1}, x^{k}-y^{k}\right) .
\end{aligned}
$$

These syzygies satisfy the relation $x R_{k, 1}+y R_{k, 2}+z R_{k, 3}=0$ and so that $C_{2 k}$ is a nearly free divisor with exponents $d_{1}=d_{2}=d_{3}=k$ and $\tau\left(C_{2 k}\right)=3 k(k-1)$. 
To prove (3) we follow as in the proof of Theorem 3.9 (3) the main difference is that $\pi_{2}$ has no ramification over $C_{2}$ and in fact $C_{4}$ is the union of four lines in general position; their preimages. If $k=2 \ell$, since $\pi_{k}=\pi_{\ell} \circ \pi_{2}$, each irreducible component is a smooth Fermat curve.

These curves, for $k \geq 3$ odd, are of positive genus and give a counterexample to the nearly free part of Conjecture 1.1](ii) (with unbounded genus and number of singularities). Furthermore, if $k \geq 5$, since $d_{1}=5 \geq 4$ then by Corollary 2.6 $C_{2 k}$ is projectively rigid. Note that it is not the case for $C_{6}$, where we find the dual to a smooth cubic; in fact, not only this special dual is nearly free, an easy computation gives the answer for the dual of a generic smooth cubic.

\section{Pencil associated to unicuspidal Rational Plane Curves}

In this section we are going to show that it is possible to construct a rational nearly free curve with singular points with more than three branches (i.e., we do not need high genus curves).

Given a curve $C \subset \mathbb{P}^{2}$, let $\pi: \widetilde{\mathbb{P}}^{2} \rightarrow \mathbb{P}^{2}$ be the minimal, (not the "embedded" minimal) resolution of singularities of $C$; let $\widetilde{C} \subset \widetilde{\mathbb{P}}^{2}$ be the strict transform of $C$, and let $\tilde{\nu}(C)=\widetilde{C} \cdot \widetilde{C}$ denote the self-intersection number of $\widetilde{C}$ on $\widetilde{\mathbb{P}}^{2}$.

A unicuspidal rational curve is a pair $(C, P)$ where $C$ is a curve and $P \in C$ satisfies $C \backslash\{P\} \cong \mathbb{A}^{1}$. We call $P$ the distinguished point of $C$. Given a unicuspidal rational curve $C \subset \mathbb{P}^{2}$ with singular point $P$, in [7, 8], D. Daigle and the last named author, were interested in the unique pencil $\Lambda_{C}$ on $\mathbb{P}^{2}$ satisfying $C \in \Lambda_{C}$ and $\operatorname{Bs}\left(\Lambda_{C}\right)=\{P\}$ where $\operatorname{Bs}\left(\Lambda_{C}\right)$ denotes the base locus of $\Lambda_{C}$ on $\mathbb{P}^{2}$.

Let $\pi_{m}: \widetilde{\mathbb{P}}_{m}^{2} \rightarrow \mathbb{P}^{2}$ be the minimal resolution of the base points of the pencil. By Bertini theorem, the singularities of the general member $C_{\text {gen }}$ of $\Lambda_{C}$ are contained in $\operatorname{Bs}\left(\Lambda_{C}\right)=\{P\}$.

For a unicuspidal rational curve $C \subset \mathbb{P}^{2}$, we show (cf. [8, Theorem 4.1]) that the general member of $\Lambda_{C}$ is a rational curve if and only if $\tilde{\nu}(C) \geq 0$. In such a case

(1) the general element $C_{\text {gen }}$ of $\Lambda_{C}$ satisfies that the weighted cluster of infinitely near points of $C_{\text {gen }}$ and $C$ are equal (see [7, Proposition 2.7]).

(2) $\Lambda_{C}$ has either 1 or 2 dicriticals, and at least one of them has degree 1. 
In view of these results, it is worth noting that all currently known unicuspidal rational curves $C \subset \mathbb{P}^{2}$ satisfy $\tilde{\nu}(C) \geq 0$, see [8, Remark 4.3] for details.

Let $C \subset \mathbb{P}^{2}$ be a unicuspidal rational curve of degree $d$ and with distinguished point $P$. In in [8, Proposition 1] it is proved $\Lambda_{C}$ is in fact the set of effective divisors $D$ of $\mathbb{P}^{2}$ such that $\operatorname{deg}(D)=d$ and $i_{P}(C, D) \geq d^{2}$. The curve $C \in \Lambda_{C}$ because $i_{P}(C, C)=\infty>d^{2}$.

The main idea here is to take the general member $C_{\text {gen }}$ of the pencil $\Lambda_{C}$ for a nonnegative curve, i.e $\tilde{\nu}(C) \geq 0$. Doing this one gets a rational cuve $C_{\text {gen }}$ whose singularities is $\operatorname{Sing}\left(C_{\text {gen }}\right)=\{P\}$ and the branches of $C_{\text {gen }}$ at $P$ is nothing else than the sum of the degrees of the dicriticals.

The clasification of unicuspidal rational plane curve with $\bar{\kappa}\left(\mathbb{P}^{2} \backslash C\right)=1$ has been started by Sh. Tsunoda [27] and finished by K. Tono [26] (see also p. 125 in [15]).

Our next example starts with $C_{49}$ with $\bar{\kappa}\left(\mathbb{P}^{2} \backslash C_{49}\right)=1$. Secondly we take the pencil $\Lambda_{C_{49}}$, and finally its general member $C_{49, g e n}$ has degree 49 and is rational nearly-free with just one singular point which has 4 branches.

The curve $C_{49}$ is given by

$$
f_{49}=\left(\left(f_{1}^{s} y+\sum_{i=2}^{s+1} a_{i} f_{1}^{s+1-i} x^{i a-a+1}\right)^{a}-f_{1}^{a s+1}\right) / x^{a-1}=0
$$

where $f_{1}=x^{4-1} z+y^{4}, a=4, s=3, a_{2}=\ldots=a_{s} \in \mathbb{C}$ and $a_{s+1} \in \mathbb{C} \backslash\{0\}$. We can e.g. $a_{2}=\ldots=a_{s}=0 \in \mathbb{C}$ and $a_{s+1}=1$. In this case, $d=a^{2} s+1=49$, and the multiplicity sequence of $\left(C_{49}, P\right)$ of the singular point $P:=[0,0,1]$ is $\left[36,12_{7}, 4_{6}\right]$. It is no-negative with $\tilde{\nu}\left(C_{49}\right)=1$.

If we consider the rational curves $C_{4}$ defined by $f_{1}=0$ (resp. $C_{13}$ defined by $\left.f_{13}:\left(f_{1}\right)^{3} y+x^{13}=0\right)$ then $i_{P}\left(C_{49}, C_{4}\right)=4 \cdot 49\left(\right.$ resp. $\left.i_{P}\left(C_{49}, C_{13}\right)=13 \cdot 49\right)$. Thus the curve $C_{13} C_{4}^{s(a-1)}$ belongs to the pencil $\Lambda_{C_{49}}$ if $s(a-1)=9$.

If we take the curve $C_{49, \text { gen }}$ defined by $f_{49, g e n}:=f_{49}+13 f_{13} f_{4}^{9}=0$. This curve is irreducible, rational and $\operatorname{Sing}\left(C_{49, g e n}\right)=\{P\}$ and the number of branches of $C_{49, g e n}$ at $P$ is 4 .

It is nearly free by using the computations with Singular [9]. A minimal resolution (2.3) for $f_{49, g e n}$ is determined by three syzygies of degrees $d_{1}=24$ and $d_{2}=d_{3}=25$. So that $\operatorname{mdr}\left(f_{49, g e n}\right)=24$. The computations yield a relation between these syzygies of multidegree $(2,1,1)$. Then $C_{49, g e n}$ is a rational nearly free curve. Let us note that a direct computation using Singular [9] of the Tjurina number of the singular point of the curve fails, but the nearly-free condition makes 
the computation possible via Theorem 2.7] $\tau\left(C_{49, g e n}\right)=(49-1)(49-24-1)+$ $24^{2}-1=1727$ which is the result in Singular using characteristic $p=1666666649$.

\section{REFERENCES}

[1] E. Artal, Sur les couples de Zariski, J. Algebraic Geom. 3 (1994), no. 2, 223-247. 3.1

[2] E. Artal and Dimca A., On fundamental groups of plane curve complements, Ann. Univ. Ferrara Sez. VII Sci. Mat. 61 (2015), no. 2, 255-262.1

[3] E. Artal and J. Carmona, Zariski pairs, fundamental groups and Alexander polynomials, J. Math. Soc. Japan 50 (1998), no. 3, 521-543. 3.1

[4] E. Artal, J.I. Cogolludo-Agustín, and J. Ortigas-Galindo, Kummer covers and braid monodromy, J. Inst. Math. Jussieu 13 (2014), no. 3, 633-670. 1, 3.1, 3.1, 3.3, 3.4, 3.5, 3.7, 3.8, (2)a, 3.2

[5] J.I. Cogolludo-Agustín, Fundamental group for some cuspidal curves, Bull. London Math. Soc. 31 (1999), no. 2, 136-142. 3.1, 3.2

[6] J.I. Cogolludo-Agustín and R. Kloosterman, Mordell-Weil groups and Zariski triples, Geometry and Arithmetic (G. Farkas C. Faber and R. de Jong, eds.), EMS Congress Reports, Europ. Math. Soc., 2012, Also available at arXiv:1111.5703 [math.AG]. 3.1, 3.2

[7] D. Daigle and A. Melle-Hernández, Linear systems of rational curves on rational surfaces, Mosc. Math. J. 12 (2012), no. 2, 261-268, 459. 4, 1

[8] _ Linear systems associated to unicuspidal rational plane curves, Osaka J. Math. 51 (2014), no. 2, 481-511. 1, 4, 4

[9] Wolfram Decker, Gert-Martin Greuel, Gerhard Pfister, and Hans Schönemann, Singular 4-0-2 - A computer algebra system for polynomial computations, http://www.singular.uni-kl.de, 2015. 1, 1, 3.2, 4,

[10] A. Dimca, Freeness versus maximal global Tjurina number for plane curves, Preprint available at arXiv:1508.04954 [math.AG], 2015. 1, 2.1, 2.3, 2.7, 2.3

[11] A. Dimca and E. Sernesi, Syzygies and logarithmic vector fields along plane curves, J. Éc. polytech. Math. 1 (2014), 247-267. 2.1

[12] A. Dimca and G. Sticlaru, Free and nearly free surfaces in $\mathbb{P}^{3}$, Preprint available at arXiv: 1507.03450v3 [math.AG], 2015.2.2

[13] —, Free divisors and rational cuspidal plane curves, Preprint available at arXiv: 1504.01242v4 [math.AG], 2015. 1, 1 2.1, 3.2, 3.2

[14] - Nearly free divisors and rational cuspidal curves, Preprint available at arXiv: 1505.00666v3 [math. AG], 2015. 1, 1.1, 1, 1.2, 1, 2.2, 2.4, 2.2, 2.5, 2.6

[15] J. Fernández de Bobadilla, I. Luengo-Velasco, A. Melle-Hernández, and A. Némethi, On rational cuspidal projective plane curves, Proc. London Math. Soc. (3) 92 (2006), no. 1, 99-138. 1, 目

[16] H. Flenner and M.G. Zaĭdenberg, Rational cuspidal plane curves of type $(d, d-3)$, Math. Nachr. 210 (2000), 93-110. 2.3 
[17] A. Hirano, Construction of plane curves with cusps, Saitama Math. J. 10 (1992), 21-24. 3.1, 3.2

[18] N. Lindner, Cuspidal plane curves of degree 12 and their Alexander polynomials, Master's thesis, Humboldt Universität zu Berlin, Berlin, 2012. 3.1, 3.2

[19] P. Orlik and H. Terao, Arrangements of hyperplanes, Grundlehren der Mathematischen Wissenschaften, vol. 300, Springer-Verlag, Berlin, 1992. 3.10

[20] A.A. du Plessis and C.T.C. Wall, Application of the theory of the discriminant to highly singular plane curves, Math. Proc. Cambridge Philos. Soc. 126 (1999), no. 2, 259-266. 11, 2.3

[21] K. Saito, Theory of logarithmic differential forms and logarithmic vector fields, J. Fac. Sci. Univ. Tokyo Sect. IA Math. 27 (1980), no. 2, 265-291.1

[22] H. Schenck and Ş.O. Tohăneanu, Freeness of conic-line arrangements in $\mathbb{P}^{2}$, Comment. Math. Helv. 84 (2009), no. 2, 235-258. 3.10

[23] E. Sernesi, The local cohomology of the Jacobian ring, Doc. Math. 19 (2014), 541-565. 1, 2.1

[24] W.A. Stein et al., Sage Mathematics Software (Version 6.7), The Sage Development Team, 2015, http://www . sagemath.org. 1

[25] G. Sticlaru, Invariants and rigidity of projective hypersurfaces, Bull. Math. Soc. Sci. Math. Roumanie (N.S.) 58(106) (2015), no. 1, 103-116.2.1

[26] K. Tono, On rational unicuspidal plane curves with $\bar{\kappa}=1$, Newton polyhedra and singularities (Japanese) (Kyoto, 2001), RIMS Kokyuroku, vol. 1233, Kyoto University, 10 2001,

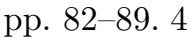

[27] Sh. Tsunoda, The complements of projective plane curves, Commutative Algebra and Algebraic Geometry, RIMS Kokyuroku, vol. 446, Kyoto University, 12 1981, pp. 48-56. 4

[28] A.M. Uludă̆, More Zariski pairs and finite fundamental groups of curve complements, Manuscripta Math. 106 (2001), no. 3, 271-277. 3.1, 3.1

[29] J. Vallès, Free divisors in a pencil of curves, Preprint available at arXiv:1502.02416v1 [math.AG], 2015. 1

iUma, Departamento de Matemáticas, Facultad de Ciencias, Universidad de Zaragoza, C/ Pedro Cerbuna 12, 50009 Zaragoza, SPAin

E-mail address: artal@unizar.es

iCMAT (CSIC-UAM-UC3M-UCM), Departamento de Álgebra, FaCultad de Ciencias Matemáticas, Universidad Complutense, 28040 Madrid, SPAin

E-mail address: leire.gg@gmail.com, iluengo@mat.ucm.es, amelle@mat.ucm.es 